\title{
Features of Electroconductivity of $\gamma$-Irradiated Composites in Heating-Cooling Conditions
}

\author{
Nabi Shamshad Aliyev, Musafir Mazahir Guliyev, Arif Musa Maharramov, \\ Rafiga Soltan Ismayilova
}

Institute of Radiation Problems of Azerbaijan National Academy of Sciences, Baku, Azerbaijan

Email address:

rafiqa55@ramblerl.ru (R. S. Ismayilova)

\section{To cite this article:}

Nabi Shamshad Aliyev, Musafir Mazahir Guliyev, Arif Musa Maharramov, Rafiga Soltan Ismayilova. Features of Electroconductivity of $\gamma$ Irradiated Composites in Heating-Cooling Conditions. American Journal of Physics and Applications. Vol. 3, No. 2, 2015, pp. 15-20. doi: 10.11648/j.ajpa.20150302.11

\begin{abstract}
It has been studied the behavior of temperature dependence of volume electroconductivity $\sigma$ on direct current (DC) of composite medium of high-density polyethylene $/ \alpha-\mathrm{Fe}_{2} \mathrm{O}_{3}$ in the field of $\mathrm{T}=20-135^{0} \mathrm{C}$ within heating-cooling conditions before and after $\gamma$-irradiation at dose up to $500 \mathrm{kGy}$. At $\lg \sigma(\mathrm{T})$ dependence of unirradiated composites while heating at low and high temperatures besides linear sections, it is observed the region of sharp decrease at $50-80^{\circ} \mathrm{C}$, associated with dipole orientation processes of adsorbed water molecules. At $\lg \sigma(\mathrm{T})$ dependence of composite samples irradiated at $\mathrm{D}=100 \mathrm{kGy}$ there is not any region of sharp decrease $\sigma$. It is shown that after $\gamma$-irradiation by dose $\mathrm{D}=500 \mathrm{kGy}$ the second maximum appears on the curve of forward trace of the composite HDPE $+10 \% \alpha-\mathrm{Fe}_{2} \mathrm{O}_{3}$ at $\mathrm{T}=80^{\circ} \mathrm{C}$, which can be the result of change in the state of quasi-Fermi level. Behavior of $\lg \sigma(\mathrm{T})$ function at heating-cooling is explained by the "asymmetry" effect of electrically active defects. It is shown that the increase in volume conductivity after $\gamma$-irradiation is weakly expressed.
\end{abstract}

Keywords: Composite, Heating-Cooling, Percolation, Electroconductivity, $\gamma$-Irradiation, High Density Polyethylene

\section{Introduction}

The widespread use of polymer dielectrics and electroactive polymer composites (EAPC) with micro- and nano-size inorganic (ferroelectric piezo- and pyroelectric ceramic materials, semiconductors, metal oxides, etc.) fillers as high-voltage electrical insulating materials in nuclear power plants and reactors, in cable technology [1-3], as well as electroactive elements (thin-film transistors, organic photocells, light-emitting diodes, touch panels, solar cells, random access memories and copiers, etc.) in appliances and modern electronics devices [4-7], including the power supply systems of satellites and spacecraft, in which there is always nuclear radiation, is caused by a number of unusual and perspective properties in the applied relation. It should be noted that the electroconductivity of composites is the dominant parameter, in which these devices operate. Therefore active studies are conducted, dedicated to the obtain and electroconductivity of EAPC. However, they have lower conductivity compared to metals and are less stable, especially under the influence of ionizing radiation, temperature and other environmental factors have a major influence on the functional characteristics of the composites. These defects significantly limit their practical application. In particular, there is no detailed information about the process of electrotransfer in the structure at different voltages and frequencies of the applied electric field, temperature dependence of the electro-physical characteristics in terms of heating - cooling, etc. Despite intensive study of the properties of composite structures, it has not yet been revealed the mechanism of carrier trapping, their retention and emission from localized states, defining parameters of certain effects, not fully understood the role of the phase boundary polymer-filler and the processes occurring at the boundary of electrode with the sample (at the interface), etc. Currently, there is no theory that allows from a single physical concepts to describe the features of the electrical properties of heterogeneous materials in a wide temperature and frequency ranges. In this regard, currently preference is given to experimental methods of studying features of interface and electrical characteristics of composite materials.

For the development of a new class of analytical devices it is necessary to develop new "smart" materials - EAPC in which the insulating polymer matrix comprises a micro - or 
nano-particles of semiconductor or metal oxides. In our previous work [8], we reported the results of the experimental study of electrophysical properties of the composite system of high density polyethylene HDPE/ $\alpha$ $\mathrm{Fe}_{2} \mathrm{O}_{3}$. It was shown that the dependence of the real part of the permittivity $\left(\varepsilon^{\prime}\right)$, dielectric $\operatorname{loss}(\operatorname{tg} \delta)$ and electrical conductivity $(\sigma)$ on direct current $(\mathrm{dc})$ on temperature of composites of $\mathrm{HDPE} / \alpha-\mathrm{Fe}_{2} \mathrm{O}_{3}$ are nonmonotonic, change in the electroconductivity depending on concentration of filler is threshold and for these composites, there are at least two mechanisms of conduction.

Radiation-induced behavior of EAPC properties is an important aspect to which you should pay special attention within the development of new EAPC working under the influence of nuclear radiation (since these materials are successfully used as an anti-damage blanket of a nuclear reactor).

The aim of this work is the experimental study of the electroconductivity of the generated model macro systems depending on the temperature, the concentration of the filler and the absorbed dose (D) of $\gamma$ - radiation in heating-cooling. We believe that the presented results of the experimental study of temperature, concentration and dose dependences of the electrical conductivity of polymer composites of $\mathrm{HDPE} / \alpha-\mathrm{Fe}_{2} \mathrm{O}_{3}$ in constant electric field will provide new information on the general or specific aspects of the conduction mechanisms as polymers and composite microstructures based on them.

\section{Experimental}

As a dielectric (matrix) it was chosen powdered high density polyethylene (HDPE), brand 20806-024, with average molecular weight 95,000 , degree of crystallinity $52 \%$, melting temperature of $130^{\circ} \mathrm{C}$ and density of -958 $\mathrm{kg} / \mathrm{m}^{3}$. Selection of HDPE as a matrix was dictated by mainly good dielectric properties and technological effectiveness of the material.

While manufacturing the film samples of unfilled HDPE and $\mathrm{HDPE} / \alpha-\mathrm{Fe}_{2} \mathrm{O}_{3}$ composites, the following scheme has been applied:

-obtain of $\alpha-\mathrm{Fe}_{2} \mathrm{O}_{3}$ powders with particle sizes less than 30 microns by sifting;

- mixing powdered $\alpha-\mathrm{Fe}_{2} \mathrm{O}_{3}$ with $\mathrm{HDPE}$ powder in a porcelain mortar;

-compression of homogenous mixture of powder components in hydraulic press with heated plates at a pressure of $15 \mathrm{MPa}$ with soaking at $150^{\circ} \mathrm{C}$ for $5 \mathrm{~min}$ and obtain of composite samples in the form of discs with diameter of $20 \mathrm{~mm}$ and thickness $140 \div 180$ microns;

- for the purpose of providing reliable electrical contact between the sample and the electrodes from stainless steel, the compression on both working surfaces of samples of electrodes from thin aluminum foil of thickness 7 microns with aftercooling in mixture of water-ice (annealing mode).

The samples of composites had connectivity type 0-3[8]. All the concentrations (C) shown in the work are volumetric.
The study of the materials were conducted by twoelectrode scheme at the temperature range of $20-135^{\circ} \mathrm{C}$ using a special shielded and grounded heated measuring cell of "sandwich" type, used for heating and cooling of the samples, having system of measuring and potential electrodes with diameters of 10 and $20 \mathrm{~mm}$, respectively. Samples were placed in the measuring cell with clamping electrodes from stainless steel. The sample temperature was controlled by using a thermocouple copper-constantan. The electroconductivity of the samples was measured with a thermometer E6-13A. The value of electroconductivity $\sigma$ was calculated from the relationship:

$$
\sigma=\frac{1}{R} \cdot \frac{h}{S}
$$

where R (ohm) - resistance of the sample measured by teraohmmeter, h- thickness of sample, $\mathrm{S}\left(\mathrm{M}^{2}\right)$ - the surface of the sample. The rate of temperature change for all samples in the cooling and heating mode was maintained constantly and was $3^{0} \mathrm{C} / \mathrm{min}$. Before the measurements, all samples were subjected to "electrocleaning" within 60 minutes in a constant electric field with the intensity of $\mathrm{E}=150 \mathrm{kV} / \mathrm{m}$. During this time, constant value of open-end current is set, and therefore the cause of the error was eliminated, associated with the contact phenomena at the boundary of sample-electrode. Film samples of the composites were subjected to $\gamma$-radiation at room temperature on the device with a radiation source ${ }^{60} \mathrm{Co}$. Absorbed dose rate is $3,3 \cdot 10^{3} \mathrm{~Gy} / \mathrm{h}$.

\section{Results and Discussion}

The results of studies of concentration, temperature and dose dependences of the electroconductivity on direct current of HDPE samples and composites of $\mathrm{HDPE}+\alpha-\mathrm{Fe}_{2} \mathrm{O}_{3}$ are shown in figure 1-3.

Before proceeding to the consideration of these dependencies, it should be noted the influence of absorbed dose of $\gamma$-irradiation on the electroconductivity of this system (not as a separate figure). Two areas are allocated for the concentration dependence of conductivity: comparatively rapid growth $\sigma$ up to $10 \%$ and flat field of $\sigma$ increase up to $50 \%$. With increase in concentration of $\alpha-\mathrm{Fe}_{2} \mathrm{O}_{3} \sigma$ increased from $3 \cdot 10^{-14}(\mathrm{Ohm} \cdot \mathrm{m})^{-1}$ for HDPE up to $1,2 \cdot 10^{-6}(\mathrm{Ohm} \cdot \mathrm{m})^{-1}$ for $\mathrm{HDPE}+50 \% \alpha-\mathrm{Fe}_{2} \mathrm{O}_{3}$. Influence of $\gamma$ - radiation on the test samples up to $500 \mathrm{kGy}$ dose leads to a slight increase in the value of $\sigma$ (increase of $\sigma$ after irradiation is weakly expressed), does not affect the function of the dependence $\sigma$ $=\mathrm{f}(\mathrm{C})$, that curve of this function is located on the top, practically parallel curve to $\gamma$ - irradiation and does not change the percolation threshold. A slight increase in the bulk conductivity of HDPE and composites based on it after $\gamma$ irradiation can be explained as follows: at prolonged radiation influence the related charges, usually manage to recombine (samples are not polarized under the influence of $\gamma$-radiation), and relaxation process of injected carriers occurs 
for a short time. So, $\sigma$ is caused mainly by the free charges.

Figure 1 shows the results of measuring temperature dependence of the electroconductivity of $\mathrm{HDPE}+\alpha-\mathrm{Fe}_{2} \mathrm{O}_{3}$ samples, presented in the coordinates $\lg \sigma-\mathrm{T}$, before (a) and after (b) the influence of $\gamma$ - irradiation dose $100 \mathrm{kGy}$. From the figure it can be seen that in both cases the function $\lg \sigma=$ $\mathrm{f}(\mathrm{T})$ has a complex form with characteristic "breaks", associated with phase transitions. Conductivity of the system HDPE $+\alpha-\mathrm{Fe}_{2} \mathrm{O}_{3}$ is more than the original matrix HDPE in the all studied temperature range. All samples have low conductivity $\left(\sigma \approx 10^{-13}-10^{-9} \mathrm{Ohm}^{-1} \mathrm{~m}^{-1}\right)$, characteristic for dielectrics. With increasing temperature, the conductivity of the nonirradiated samples of composites increases by 2-3 order. For comparison, the same figure shows the temperature dependence of pure HDPE. By increasing the temperature up to $70^{\circ} \mathrm{C} \sigma$ of HDPE remains practically constant, then in the area (area of softening of the matrix) is slightly increased, and by the end of the temperature range $\left(100-135^{\circ} \mathrm{C}\right)$ it stabilizes. For composites of $\mathrm{HDPE}+\alpha-$ $\mathrm{Fe}_{2} \mathrm{O}_{3}$ system it is observed a very different conduct of $\lg \sigma=f(T)$ function. With increasing temperature, the composite conductivity increases, reaches a maximum (I area) then begins to decrease with further increase of temperature and reaches minimum at $80^{\circ} \mathrm{K}$ (II area), and then increases again until the end of the temperature scale (III area), i.e. in the graphs of the temperature dependence $\lg \sigma$ it is observed three sites. Reduction of $\sigma$ of composite samples at the range $\left(50-80^{0}\right) \mathrm{C}$ occurs by approximately $1,5 \div 2$ order and practically reaches the initial conductivity. The presence of the peak at $70^{\circ} \mathrm{C}$ can be related to dipolar orientation of adsorbed water molecules. We come to this assumption by the fact that: this peak is observed at higher temperatures (see figure 2) and is not observed during the reverse course of conductivity, i.e. at decreasing temperature. So, with increasing of temperature there occurs desorption of water molecules and conductivity, associated with $\mathrm{H}_{2} \mathrm{O}$ dipoles decreases. Monotonic increase of composite conductivity with increasing temperature in the I and III areas, practically linearly, shows the exponential nature of the dependence of $\sigma$ (T) function and the existence of two areas of freezing impurity levels in these samples, from which charge carriers (electrons) are released in the case of heating. Increase of $\sigma$ in the third area, probably is conditioned by both an increase in segmental mobility of the polymer matrix and carriers of current, supplied with $\alpha-\mathrm{Fe}_{2} \mathrm{O}_{3}$ particles [8]. Hightemperature area (area of inherent conductivity) of the conductivity is located between the temperatures $80-135^{\circ} \mathrm{C}$. At these temperatures inherent conductivity for composites increases by the order 5, 6 and 7, respectively. In the lowtemperature impurity area the conductivity of $\sigma$ increased by order 1.5-3.

Influence of $\gamma$-radiation on the electro conductivity of $\mathrm{HDPE}+\alpha-\mathrm{Fe}_{2} \mathrm{O}_{3}$ system was investigated at the range of absorbed dose up to $0-500 \mathrm{kGy}$. The results of these studies are shown in Figure 1b. The figure shows that the temperature dependence of the conductivity of $\sigma$ of all samples after the influence of ionizing radiation is undergoing major changes, namely: 1) for pure HDPE, unlike no irradiated sample with increasing temperature $\sigma$ increases, reaches a maximum at $95^{\circ} \mathrm{C}$ and begins to decrease at further increase of temperature up to $115^{\circ} \mathrm{C}$ and then gets stabilized; 2) with increasing temperature (temperature dependence) conductivity of $\mathrm{HDPE}+10 \% \alpha-\mathrm{Fe}_{2} \mathrm{O}_{3}$ composites is close to the corresponding dependence $\lg \sigma=\mathrm{f}(\mathrm{T})$ for these composites before $\gamma$-irradiation, but the position of the maximum $\sigma$ is shifted to lower temperatures of $10^{\circ}$ and minimum to considerably high temperatures; 3) after $\gamma$ irradiation the position of the maximum of conductivity of HDPE $+20 \% \alpha-\mathrm{Fe}_{2} \mathrm{O}_{3}$ composite is shifted to higher temperatures of $25^{\circ}$, and then, until the end of the temperature scale it is observed almost stable site of $\sigma$ values The figure also shows that after $\gamma$ - irradiation the area of sharp slump of conductivity of composites disappears in the area of $70-80^{\circ} \mathrm{C}$.
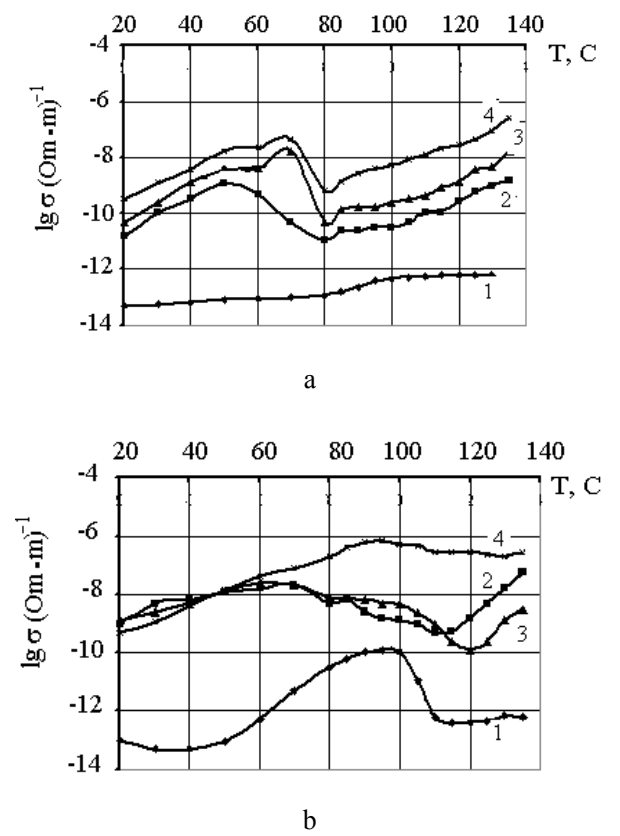

Figure 1. Temperature dependence of the HDPE electroconductivity, containing 0(1), 5 (2), 10(3), 20\% (4) of $\alpha-\mathrm{Fe}_{2} \mathrm{O}_{3}$ before (a) and after (b) $\gamma$ irradiation $100 \mathrm{k} G$.

We will consider the possible reasons causing the abovementioned changes in conduct of the function $\lg \sigma=\mathrm{f}(\mathrm{T})$ after $\gamma$ - irradiation. It is known that [9-13], within the accumulation of dose it is becoming increasingly important firstly the radiation-chemical effects (formation of radical and molecular products of radiolysis which leads to the change of energy distribution of traps due to appearance of radiation-generated traps) and then deep chemical conversions in polymer (destruction, cross-linking, amorphization, gas emission, etc.) leading to the change in the nature of polymer and inconvertible dose effect. In the irradiation process there also occurs the crystallite fracture (reduction of their size, increase of the efficient boundary surface), i.e. decrease of general rate of crystallinity, relocation of traps in depth: decrease in density of interphase deep traps, but simultaneously the concentration of shallow 
electron traps, associated with carbonyl $(\mathrm{C}=\mathrm{O})$ groups (oxidation product) or with radiation-induced defects increases. Capture of charges, generated after $\gamma$-radiation, occurs at interphase boundaries of amorphous and crystal phases [14]. Carbonyl and various peroxide groups formed during $\gamma$-irradiation, provide small and interphase levels of capture lying at depths of up to $1 \mathrm{eV}$. Within $\gamma$-irradiation the accumulation of the volume electric charge in HDPE correlates with formation and accumulation of peroxide radicals which as electron acceptors determine the whole process of charge accumulation. Therefore most authors consider that the dose effect, i.e. the influence of preliminary irradiation on electroconductivity of the composites is due to, most likely, not change of the spectrum of molecular motions but to the accumulation in the irradiated material of stabilized charge carriers and to a lesser extent, radical and molecular products of radiolysis acting as a center of capture. The influence mechanism of dose on charge carrier transfer is connected with the change in the spectrum of molecular relaxation. Dominating in the initial stages at low irradiation doses, the degradation of macromolecules mainly weakens the intermolecular interaction and initiates the secondary crystallization of the surface layer of films during which process formed at radiolysis of charge carriers, are captured by concentrated low-energy traps near the surface. At further growth of dose on the surface layer, the accumulation processes prevail the dislocation and formation of micro cracks conditioning the relaxation of captured charges [15].

Comparison of the temperature conduct of $\sigma$ composites shows that after $\gamma$-irradiation (figure 1, b, curves 2,3 and 4) for $\mathrm{HDPE}+5 \% \alpha-\mathrm{Fe}_{2} \mathrm{O}_{3}$ and $\mathrm{HDPE}+10 \% \alpha-\mathrm{Fe}_{2} \mathrm{O}_{3}$ systems the rate of decrease of $\sigma$ is more with temperature increase than in case of HDPE $+20 \% \alpha-\mathrm{Fe}_{2} \mathrm{O}_{3}$, which is consistent with the arguments on the presence of clusters of in the polymer matrix. Within the range of $20-60^{\circ} \mathrm{C}$ the curves of $\sigma$ growth merge into a single straight line after irradiation.

Figure 2a-c shows the curves of temperature dependence of $\sigma$ under heating-cooling conditions in coordinates of $\lg \sigma=$ $\mathrm{f}(\mathrm{T})$ for HDPE $+10 \% \alpha-\mathrm{Fe}_{2} \mathrm{O}_{3}$ composites before (a) and after (b-v) $\gamma$ - irradiation at dose of 100 and $500 \mathrm{kGy}$, respectively: curve 1 - direct (increase of temperature), 2- reverse (decrease of temperature). Experiments have shown that the difference between direct and reverse values of $\sigma$, that is "range" of electroconductivity at room temperature of composite medium of $\mathrm{HDPE}+\alpha-\mathrm{Fe}_{2} \mathrm{O}_{3}$ is not high or coincide actually with the reverse curve for the same composites. Therefore figure 2 shows the maximum obtained experimental ranges for $\mathrm{HDPE}+10 \% \alpha-\mathrm{Fe}_{2} \mathrm{O}_{3}$. It can be seen from the presented dependences that breaking of cooling of samples leads to monotone decline in conductivity, which increases with decrease of temperature. For irradiated composite the conductivity of reverse motion of $\lg \sigma(\mathrm{T})$ (fiqure.2, b-c) is higher up to $100^{\circ} \mathrm{C}$, than forward trace, after which the curves of forward and reverse motions cross and difference between $\sigma$ values intensifies: At $\mathrm{T}<105^{\circ} \mathrm{C}$ values of $\sigma$ for the same temperature is lower within cooling than heating of sample. In case of no irradiated $(D=0)$ composite of $\mathrm{HDPE}+10 \% \alpha-\mathrm{Fe}_{2} \mathrm{O}_{3}$ intersection point of forward and reverse motions is shifted to lower temperature of $30^{\circ} \mathrm{C}$, that connected with the presence of the region of sharp decrease in conduction at temperature of $70^{\circ} \mathrm{C}$ in these samples. From comparison of figure $2, \mathrm{~b}$ and $\mathrm{v}$ it is seen that exposure dose of absorption influence differently on the curve of forward motion. We have already discussed the conduct of graph $\lg \sigma$ $=\mathrm{f}(\mathrm{T})$ at $\mathrm{D}=0$ and $\mathrm{D}=100 \mathrm{kGy}$. At $\mathrm{D}=500 \mathrm{kGy}$ the second maximum appears on the curve of forward motion at $80^{\circ} \mathrm{C}$, but the reverse branches practically don't change. We assume that appearance of the second maximum at $80^{\circ} \mathrm{C}$ on the forward branches after $\gamma$-irradiation of dose $\mathrm{D}=500 \mathrm{kGy}$ can be the result of change in position of quasi-Fermi level (reallocation of traps occurs in depth and the concentration of deep traps increases, the release of the charges from which occurs at higher temperatures) and the observed peak is connected with a different group of trap states at the crystal boundary - amorphous phase.

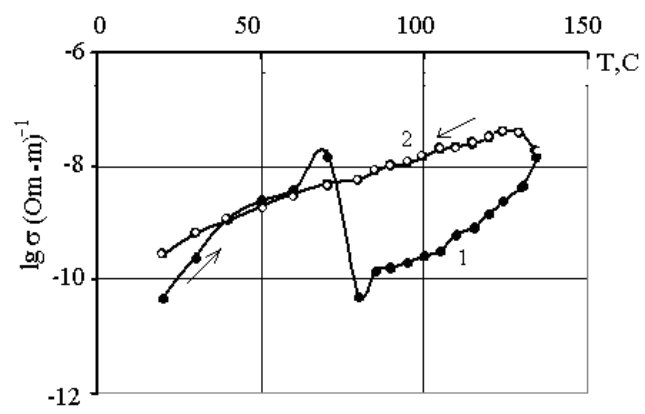

a

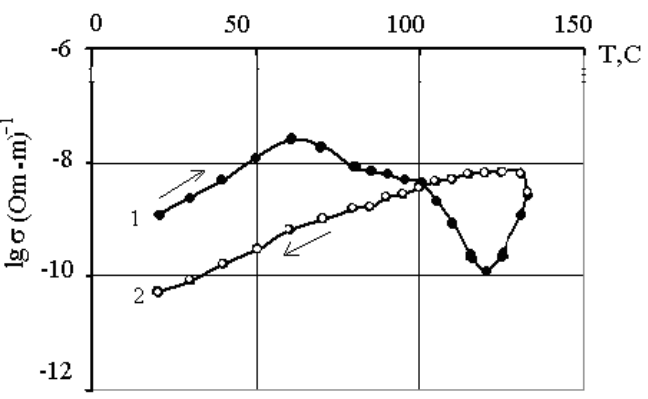

b

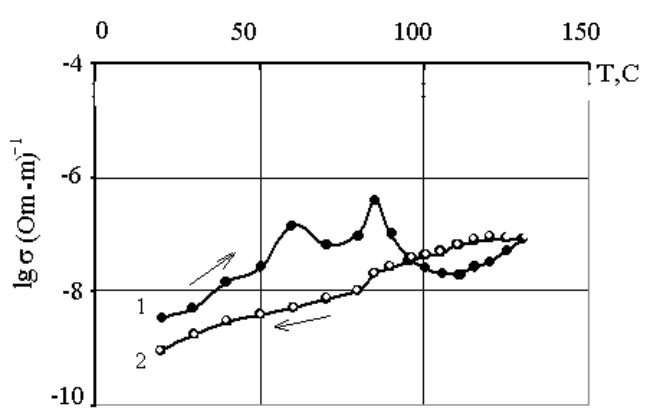

$\mathrm{c}$

Figure 2. The effect of dose of $\gamma$-irradiation on the temperature dependence of the electroconductivity of $\mathrm{HDPE}+10 \% \alpha-\mathrm{Fe}_{2} \mathrm{O}_{3}: a-D=0 ; b-D=$ $100 k G y ; c-D=500 k G y$. 1 - heating; 2 - cooling. 
According [16] to the conduct of function of $\sigma(T)$ at heating-cooling it can be explained in two ways: either the reconstructing structure of fractal chains near the fusion point, or "asymmetry" effects of temperature evolution of electrically active defects at heating-cooling. The lack of clearly expressed hysteretic processes for composite medium $\mathrm{HDPE}+\alpha-\mathrm{Fe}_{2} \mathrm{O}_{3}$ gives reason to consider that the main mechanism in this case is the effect of "asymmetry" of the temperature evolution of electrically active defects. The value of conductivity of samples in heating-cooling gets new value (composite material changes to a new state with decreased conductivity), continuing for some time (at least for ten days). Such conduct of $\mathrm{HDPE}+10 \% \alpha-\mathrm{Fe}_{2} \mathrm{O}_{3}$ composites, is apparently, connected with thermally stimulated processes of re-allocation of charge at boundary of microparticle - polymer matrix and the appearance of metastable states of the medium. By the results of measurements it has been estimated the temperature variations of the conductivity value. The decrease of conductivity with temperature change was approximately 24 and 3.7 times during one cycle of heating-cooling after $\gamma$ irradiation at doses of 100 and $500 \mathrm{kGr}$, respectively.

Figure 3 shows typical curves of the function $\sigma=f(D)$. Electrical conductivity is subjected to the greatest change in direct current of composite samples with volumetric content of the filler $\alpha-\mathrm{Fe} 2 \mathrm{O} 35$ and $10 \%$, respectively. The electroconductivity of the samples with growth of $\mathrm{D}$ reaches maximum at $\mathrm{D}=200 \mathrm{kGy}$ and $100 \mathrm{kGy}$, respectively. Subsequent growth of the absorption dose leads to reduction of $\sigma$ of $\mathrm{HDPE}+5 \% \alpha-\mathrm{Fe}_{2} \mathrm{O}_{3}$ composites, and in the case of $\mathrm{HDPE}+10 \% \alpha-\mathrm{Fe}_{2} \mathrm{O}_{3}$ composite the electroconductivity, decreasing at $300 \mathrm{kGy}$, reaches minimum and then increases again. The electroconductivity of pure HDPE and HDPE $+20 \%$ $\alpha-\mathrm{Fe}_{2} \mathrm{O}_{3}$ composites in the mentioned dose range remains almost constant. It is seen that the filling of HDPE up to $20 \%$ $\alpha-\mathrm{Fe}_{2} \mathrm{O}_{3}$ leads to increase in the electroconductivity from $3,0 \cdot 10^{-14}(\mathrm{Ohm} \cdot \mathrm{m})^{-1}$ to $3,5 \cdot 10^{-10}(\mathrm{Ohm} \cdot \mathrm{m})^{-1}$. It should be noted that, in general, the absorbed dose effects weakly to the dose dependence of the studied samples (except from HDPE $+5 \%$ $\alpha-\mathrm{Fe}_{2} \mathrm{O}_{3}$ ). We assume that some increase of electrical conductivity observed at $\gamma$-irradiation up to $200 \mathrm{kGy}$, is caused by ionization. Ionization supplies capability of displacement of the charges (free electrons and positive ions) which can participate in the process of conductivity. According to the authors [17], dose effects are direct consequence of formation of free radicals and their prorecombinations interconnected with the formation of the characteristic post-radiation changes in the structure of the polymer. Besides it, some increase observed within $\gamma$ irradiation in electric conductivity of the studied samples, can be associated with the processes of cross-linking of polymer chains, leading to the formation of three-dimensional structure, and its further decrease with the process of oxidative decomposition.

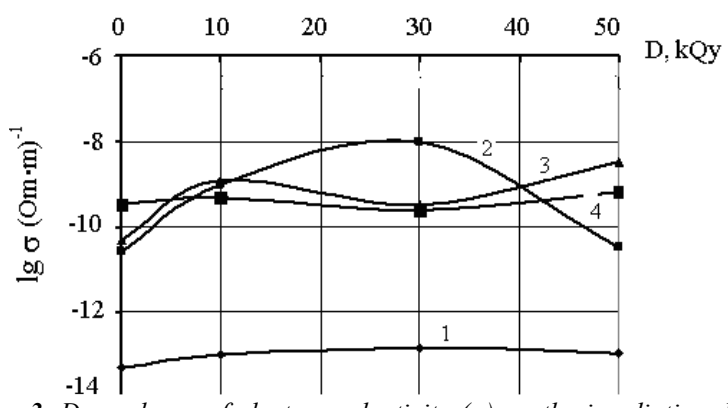

Figure 3. Dependence of electroconductivity $(\sigma)$ on the irradiation dose of $\mathrm{HDPE}+\alpha \mathrm{Fe}_{2} \mathrm{O}_{3}$ composites: 1-pure HDPE, 2- $\mathrm{HDPE}+5 \% \alpha-\mathrm{Fe}_{2} \mathrm{O}_{3}, 3-$ $\mathrm{HDPE}+10 \% \alpha-\mathrm{Fe}_{2} \mathrm{O}_{3}, 4-\mathrm{HDPE}+20 \% \alpha-\mathrm{Fe}_{2} \mathrm{O}_{3}$

Authors [18] claim that, the electroconductivity of polyethylene matrix is caused by charge carriers which appear at $\gamma$-irradiation, but not by the charges, injected from electrodes. With increasing of irradiation doses, the concentration of trapped electrons also increases: the greater the irradiation dose, the greater the number of charge carriers. As already mentioned the growth of irradiation dose leads to reduction in relation of crystal phase to amorphous and, thereby, causes the reduction of the interfacial boundary area. Consequently, the density of concentration of deep traps is reduced on the interface boundary.

It should be noted that the presented experimental data don't allow making a final conclusion yet about the specific mechanism of electroconductivity in $\gamma$-modified composite materials. The problems relating to the definition of the transport mechanism and trapping of charge carriers, temperature, frequency and dose- dependent parameters, the role of the occurring on the interface in such materials requires further investigations.

\section{Conclusions}

1. It is established that $\gamma$-irradiation significantly influences on the conduct of curves of $\lg \sigma(\mathrm{T})$ at heating and practically doesn't influence on the curve of reverse motion.

2. Relatively sharp decay of the electroconductivity of non-irradiated $\mathrm{HDPE}+\alpha-\mathrm{Fe}_{2} \mathrm{O}_{3}$ composites on the curve of forward motion is caused by desorption of $\mathrm{H}_{2} \mathrm{O}$ molecules.

3. It is established that for HDPE composites with concentration of $\alpha-\mathrm{Fe}_{2} \mathrm{O}_{3} 5,10$ and $20 \%$ at $\mathrm{T}<105^{\circ} \mathrm{C}$ the values of $\sigma$ for the same temperatures are less at cooling, than heating.

\section{References}

[1] Tanaka T. Dielectric nanocomposites with insulating poperties. IEEE Trans.Diel.Electr.Insul.2005, v.12, No5, p.914-918

[2] Ueno K., Uda I. and Tada S. Radiation - Crosslinked polyethylene for wire and cable applications. Radiat.Phys.Chem.1991, v.37, No1,p.89-91 
[3] Gefle O. S., Lebedev S.M., Tkachenko S. N. The conduct of polymer composite materials with the filler from ferroelectric ceramics in electric field. Bulletin of the Tomsk Polytechnic University. 2005 v.38, №4, .p.64-68

[4] Lin M.M., Kim H.H., Muhammed M., Kim D.K. Iron oxide based nanomagnets in nano medicine: fabrication and applications. Nano Rev.2010, 1: 4883

[5] Njugun J., Peilichowski K. Polymer nanocomposites for aerospace applications: fabrication Adv.Eng. Mater. 2004, No6, p.193-210

[6] Borsenberger P.M., Weiss D.S. Organic photoreceptors for xerography (Marsel Dekker, New York, 1993).

[7] Krishnamoorti R. Advanced Polymer-Nanocomposites: Novel Properties and Applications Chem. Mater., 2007, v.19, p.27362751

[8] Aliyev N. Sh., Maharramov A.M., Guliyev M. M., Ismayilova R. S. Electrophysical properties of the composite system of high-density polyethylene $/ \alpha-\mathrm{Fe}_{2} \mathrm{O}_{3}$ Perspective materials, 2014, No.10, p.22-27

[9] Vannikov A.V Matveev V.K, Sichkar V.P, Tyutnev A.P. Radiation effects in polymers. Electrical properties. Nauka, Moscow, 1982, 269p.

[10] Tyutnev A.P Vannikov F.V, Mingaliev G.S, Saenko V.S. Electrical phenomena during irradiation of polymers. Moscow, Energoatomizdat, 1985, 176p.
[11] Prokopyev O.V., Rozno A.G. and Gromov V.V. Structural defects in formation on volume charge in irradiated polyethylene. Radiat. Phys. Chem. 1989, v.33, No3, pp.191196

[12] Gromov V.V Physicochemical processes in the electrization of dielectrics. JPC 2005, v.79, №1, p.121-125

[13] Maharramov A.M Structure and radiation-modification of electret, piezoelectric properties of polymer composites. Baku, Science, 2001, 327p.

[14] Kuliev M.M., Ismayilova R.S. The gamma-radiation effect on the spectrum of thermally stimulated current in polyethylene of high density. Surface Engineering and Appl. Electro. Chem. 2010, v.46, No5, pp.447-451

[15] Pinchuk L.S, Koretskaya L.S, Kvarchov A.G, Shapavalov V.A, Alexandrova T.I Goldade V.A. Investigation of photoaging of polyethylene by thermally stimulated currents. Macromolecular compounds, B, 2003, v.45, №2, p.335-340

[16] Sochkov V.A, Borisov V.A. Evolution of attractor of macrosystems depending on the concentration of leading phase and temperature. JTP, 2007, v.77, ed.11, p.103-108.

[17] Tyutnev A.P, Saenko V.S Smirnov E.V, Pozhidaev U.V. Radiation electroconductive polymer during prolonged exposure. High Energy Chemistry, 2006, v.40, №5, s.364-375.

[18] Chen G., Fouracre R.A., Banford H.M.,Tedford D.J. The effects of gamma-radiation on thermally stimulated discharge current spectra in low-density polyethylene. Radiat.Phys.Chem.1991, v.37, No3, pp.523-530. 